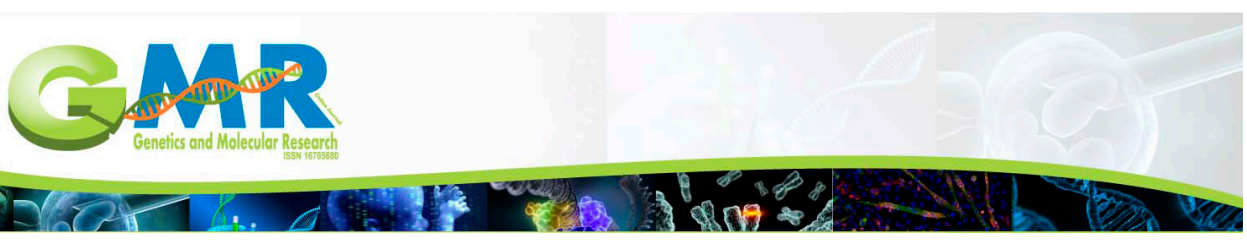

\title{
Expression of IL-21 in rats with inflammatory bowel disease
}

\author{
J.N. Mao' ${ }^{1}$, Y.S. Shen ${ }^{2}$, X.Y. Luo ${ }^{3}$ and Y.L. Zhi ${ }^{4}$ \\ ${ }^{1}$ Department of Gastroenterology, Xinxiang Central Hospital, Xinxiang, China \\ 2Department of Hand Surgery, Xinxiang Public Hospital, Xinxiang, China \\ ${ }^{3}$ Department of Gastroenterology, Beijing Friendship Hospital, \\ Capital Medical University, Beijing, China \\ ${ }^{4}$ Department of Pediatric Intensive Care Unit, Children's Hospital of Zhengzhou, \\ Zhengzhou, China \\ Corresponding author: J.N. Mao \\ E-mail: jiannamao@163.com \\ Genet. Mol. Res. 14 (4): 17322-17328 (2015) \\ Received August 22, 2015 \\ Accepted October 28, 2015 \\ Published December 16, 2015 \\ DOI http://dx.doi.org/10.4238/2015.December.16.33
}

ABSTRACT. The purpose of this study was to investigate the expression of interleukin (IL)-21 in rats with inflammatory bowel disease (IBD). Fifty adult Wistar rats were randomly divided into two groups: DSS, in which IBD was induced by giving the rats $7 \%$ DSS for seven days in their water, and a water control. Blood samples were collected and the concentration of IL21 in serum was detected by enzyme-linked immunosorbent assay. Colon tissue of rats was examined by immunohistochemical staining. Rats in the DSS group were lethargic, with matte coat color and decreasing body weight. In the DSS group, brown loss stool appeared after four days, and blood appeared in the stool along with dark red hematocele in the intestinal cavity after ten days. Rats in the control group were active and body weight increased regularly. Their stool was black and granular and the color of the intestinal canal was pink. The original body weight of all rats in both groups was similar but seven days after induction of IBD, the weight of the DSS rats dropped significantly compared to the control group $(P<0.05)$. Serum IL-21 levels were $1.37 \pm 0.43 \mathrm{pg} / \mathrm{mL}$ in the control group and $3.86 \pm 1.27$ 
$\mathrm{pg} / \mathrm{mL}$ in the DSS group $(\mathrm{P}<0.05)$. More IL-21 positive cells were detected in the intestinal mucosal epithelial cell layer and the lamina propria of the submucosa in the DSS group than in the control group. In conclusion, IL-21 is involved in the pathological process of IBD.

Key words: Interleukin-21; Inflammatory bowel disease; Rat model; Dextran sulfate sodium

\section{INTRODUCTION}

Inflammatory bowel disease (IBD) is a common chronic inflammatory disease prevalent in European and American countries as well as in China. IBD is an intestinal inflammatory disease, which includes Crohn's disease (CD) and ulcerative colitis (UC) (Coquet et al., 2007). Clinical features of IBD are repeated abdominal pain, diarrhea, and mucus and blood in stool. Patients with IBD may be treated to improve symptoms but the disease will recur in most patients and they cannot be cured completely. This results in substantial detrimental effects on quality of life of IBD patients (Ettinger et al., 2008).

Research shows that alterations in cytokines are related to IBD. Interleukin (IL)-21 expression has recently been found to be related to incidence of IBD (lannello et al., 2010). IL-21 is a member of the IL-2 family of cytokines and is located at chromosome 4q26-27. CD4+ T cells are the main source of IL-21. Research has also shown that IL-21 can modulate pathways that affect phlogosis, leading to an increase in tissue damage (Sarra et al., 2010). Interestingly, the expression of IL-21 is higher in colon tissue from patients with CD or UC (Xia and Li, 2009). High expression of IL-21 has also been found in wild-type rats with chemically-induced IBD using dextran sulfate sodium (DSS) or 2,4,6-trinitrobenzenesulfonic acid (TNBS), whereas the IL-21 receptor/Fc chimera has the effect of reducing the inflammatory reaction (Spolski and Leonard, 2008).

In order to validate the role of IL-21 in rats with IBD, we generated a chemically induced IBD model using DSS and observed the resultant levels of IL-21 in serum and in intestinal and colon tissues. These results will further our knowledge on the effect of IL-21 in IBD and could perhaps lead to new treatments for IBD.

\section{MATERIAL AND METHODS}

\section{Experimental animals}

Fifty adult Wistar rats (200-250 g) were collected from the Experimental Animal Center of Zhengzhou University (China). Rats were raised in separate cages in a sterile environment for four days in order to confirm their health. After removing food for $12 \mathrm{~h}$, the rats were weighed and randomly divided into two groups: DSS group and control group ( $\mathrm{N}=25$ each). This study was performed in strict accordance with the recommendations in the Eighth Edition of the Guide for the Care and Use of Laboratory Animals of the National Institutes of Health (Bethesda, MD, USA). The animal use protocol was reviewed and approved by the Institutional Animal Care and Use Committee (IACUC) of Xinxiang Central Hospital (China).

\section{Establishment of IBD rat model}

The IBD rat model was established using DSS (ZSGB-BIO). Rats in the DSS group were 
given water with $7 \%$ DSS for 14 days while rats in the control group were given distilled water to drink. Food intake was not modulated.

\section{Enzyme-linked immunosorbent assay (ELISA)}

After two weeks, rats in both groups were anesthetized with $4 \%$ chloral hydrate and $0.8 \mathrm{~mL}$ blood was collected retro-orbitally from each rat. Rats were sacrificed by cervical dislocation. The collected blood was stationary-cultured for $1 \mathrm{~h}$ then centrifuged at $4000 \mathrm{rpm}$ for $10 \mathrm{~min}$. Following centrifugation, the supernatant was separated and put into a $1.5-\mathrm{mL}$ tube. The detection of IL-21 in the serum was performed using an ELISA kit (Daan Gene Co., Ltd., Guangzhou, China) according to the manufacturer protocol, which included standard steps such as dilution, spotting, incubation, enzyme supplementation, coloration, and determination.

\section{Immunohistochemistry assay}

Sacrificed rats were dissected and intestinal tissue was collected and weighed. Morphological changes in the ileum and colon were noted. One part of the fresh intestinal tissue was frozen at $-80^{\circ} \mathrm{C}$ and the other part was fixed with $4 \%$ formalin. Sections were embedded with paraffin followed by removal of the paraffin and hydration. Then, sections were washed with PBS and polymer reinforcing agent (Daan Gene Co., Ltd., Guangzhou, China) followed by treatment with anti-IL-21 polyclonal antibody (Daan Gene Co., Ltd., Guangzhou, China). The sections were incubated at room temperature and 3, 3 -diaminobenzidine (DAB) color solution was used for staining. Hematoxylin was used for double staining and sections were mounted with resinene. Expression of IL-21 in colon tissue was detected with a microscope (Olympus AX80, Olympus, Tokyo, Japan). A pale brown color was indicative of positive staining for IL-21.

\section{The evaluation standard of immunohistochemistry}

The expression of IL-21 in colon tissue was divided into four classes according to its intensity: negative expression (-): the number of positive cells accounted for less than 5\%; weak positive expression (+): the number of positive cells accounted for 5-20\%; positive expression (6): the number of positive cells accounted for $20-60 \%$; and strong positive expression (7): the number of positive cells accounted for over $60 \%$.

\section{Statistical analysis}

The data was expressed as mean \pm standard deviation and analyzed using SPSS 17.0 software (SPSS Inc., Chicago, IL, USA). Group comparison was performed with a rank sum test. P $<0.05$ was considered statistically significant.

\section{RESULTS}

\section{General description of rats}

Rats in the control group were active and had bright and moist hair. Their weight increased normally and stool was black and granular throughout the entire experiment. Rats in the DSS group 
were listless and lethargic, their coat color was matte, and their body weight decreased over time. Brown loss stool appeared in the DSS group after four days. At the tenth day, blood appeared in the stool. After the dissection, we observed dark red hematoceles in the intestinal cavity in the DSS group, whereas pink intestinal cavity was observed in the control group (Table 1).

Table 1. Body weight of rats at different time points (means \pm SD, g).

\begin{tabular}{|c|c|c|c|c|c|c|c|c|}
\hline Group & Day 1 & Day 2 & Day 3 & Day 4 & Day 6 & Day 7 & Day 10 & Day 14 \\
\hline DSS group & $235.4 \pm 36.7$ & $241.6 \pm 39.3$ & $248.4 \pm 41.6$ & $242.3 \pm 39.1$ & $236.3 \pm 35.1$ & $226.0 \pm 32.8$ & $212.3 \pm 27.6$ & $\overline{193.9 \pm 23.2}$ \\
\hline Control group & $239.2 \pm 37.3$ & $243.5 \pm 39.4$ & $250.6 \pm 41.5$ & $254.8 \pm 43.2$ & $261.6 \pm 44.7$ & $274.7 \pm 46.0$ & $285.1 \pm 47.3$ & $298.4 \pm 48.7$ \\
\hline$P$ value & $>0.05$ & $>0.05$ & $>0.05$ & $>0.05$ & $>0.05$ & $<0.05$ & $<0.05$ & $<0.05$ \\
\hline
\end{tabular}

Weight difference of the DSS group was statistically significant from the control from day 7 onward $(P<0.05)$.

\section{Morphological changes of ileum and colon}

Rats in the control group had obvious colon crypt and complete epithelial structure. However, after inducing IBD in the DSS group, colitis was obvious in the blind gut and distal colon. Histopathological analysis showed that the colon crypt and epithelial structure disappeared in the DSS group, and festering, anabrosis, and apparent infiltration of inflammatory cells appeared (Figure 1).

$\mathbf{A}$

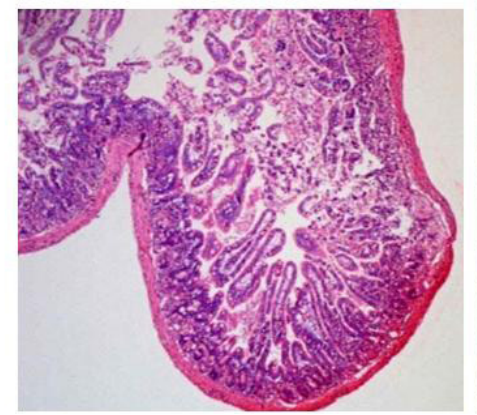

B

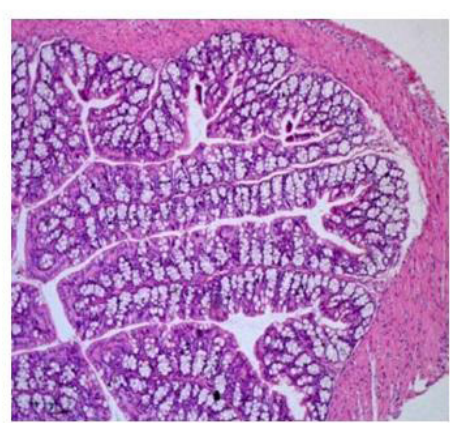

Figure 1. Morphological changes in the ileum and colon. A. After inducing IBD, colitis was seen in the blind gut and distal colon in the DSS group. Histopathological observation showed that the colon crypt and epithelial structure disappeared. Fester, anabrosis and apparent infiltration of inflammatory cells appeared. B. Rats in the control group had obvious colon crypt and complete epithelial structure (100X).

\section{Content changes of IL-21 in serum}

ELISA detection for IL-21 in serum was performed and results were $1.37 \pm 0.43$ and 3.86 $\pm 1.27 \mathrm{pg} / \mathrm{mL}$ for the control group and DSS group, respectively. The rank mean of the control and DSS groups was 3.67 and 11.23 , respectively. The $P$ value was 0.001 , indicating that the difference was statistically significant.

\section{Immunohistochemical staining of IL-21 in intestinal tissue}

Cells positive for IL-21 were observed in the mucosal epithelial cell layer of the colon and ileum in the control group. Compared to the control, more cell positive for IL-21 were detected in 
the mucosal epithelial cell layer of the colon and lamina propria of the submucosal layer of the DSS group (Figure 2).

A

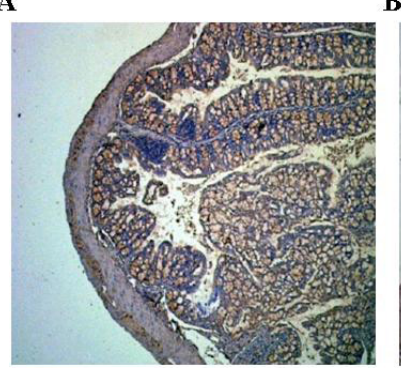

B

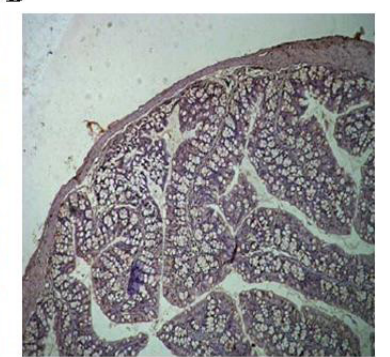

Figure 2. Immunohistochemical staining for IL-21 in intestinal tissue. A. Numerous positive cells were detected in the mucosal epithelial cell layer of the colon and lamina propria of the submucosal layer in the DSS group. B. Fewer positive cells were observed in the mucosal epithelial cell layer of the colon and ileum in the control group (400X).

\section{DISCUSSION}

Several features of IBD include inflammation of the intestinal canal and injury of intestinal mucosa. Immune disorder plays an important role in the occurrence and development of IBD. Changes in expression of different cytokines are related to IBD, including IL-21 (Fina et al., 2008). It has been shown that IL-21 can activate the inflammatory pathway in many organs, which can promote tissue damage. There is some evidence showing that overexpression of IL-21 in the intestinal tract will lead to damage. In addition, IL-21 is widely involved in the pathological processes of autoimmune diseases such as UC, CD, systemic lupus erythematosus, and rheumatoid arthritis (Monteleone et al., 2010).

Vogelzang et al. (2008) found that serum expression of IL-21 and levels of CD4+ T cells, CD8+ $T$ cells and B cells in peripheral blood were higher in UC patients compared to healthy controls and correlated with disease severity. Caruso et al. (2009) found that the expression of IL21 in CD4+ T cells, CD8+ T cells, CD20+ B cells, and CD56+ natural killer (NK) cells in peripheral blood and lamina propria from patients with UC was higher compared to healthy controls. Zhu et al. (2010) found that IL-21 played an important role in the occurrence and development of the inflammatory reaction of UC in rats, whereas rats lacking IL-21 were not affected by the inflammatory reaction. IL-21 receptor/Fc chimera had a notable effect on DSS-induced IBD in rats, producing watery diarrhea and weight reduction.

Our investigation indicated that rats in the DSS group were listless and lethargic, with matte coat color and decreasing body weight. Their stool was black and granular at beginning of this study, but brown loss stool appeared after four days of DSS treatment. After ten days of treatment with DSS, blood appeared in the stool of the rats. The sacrificed rats in the DSS group had dark red hematoceles in the intestinal cavity, indicating that oral consumption of liquid 7\% DSS successfully induced UC. The results of the ELISA showed that serum content of IL-21 in the control group was significantly less than in the DSS group $(P<0.05)$. Immunohistochemistry showed that numerous cells positive for IL-21 were detected in intestinal epithelial cells and the submucosal layer of the lamina propria of the small intestine and colon in both groups but more positive cells were observed in the DSS group than in the control group. This indicates that IL-21 is involved in the inflammatory reaction of UC induced by DSS, which is also supported by previous studies. 
The mechanism of IL-21 in the pathology of IBD is still unclear. Many reports claim that it is related to matrix metalloproteinases (MMPs), which are enzymes that can decompose all extracellular matrix content (Shichita et al., 2009). IL-21 is produced by T cells and can stimulate phoroblasts to produce MMPs, which may lead to the degeneration of mucosa. The excessive activity of MMPs has been linked to many diseases, including rheumatoid arthritis and osteochondritis (Abromson-Leeman et al., 2009). MMPs also play an important role in the homogenate degradation of $C D$ and $U C$. Studies have shown that active T cells in the lamina propria of explanted fetal intestine can stimulate phoroblasts to secrete more MMP, which activates the degradation of extracellular matrix (Snoeijs et al., 2011).

The basal expression of IL-21 in intestinal phoroblasts is necessary for signal transduction, which is initiated by IL-21; thus, phoroblasts are the potential target cell for IL-21 (Park et al., 2011). In addition, IL-21 increases the ability of phoroblasts to produce MMP-1, MMP-2, MMP-3 and MMP-9, which, together with tumor necrosis factor- $\alpha$ (TNF- $\alpha$ ), further increases the production of MMPs. Supernatant obtained from lamina propria mononuclear cells (LPMCs) from IBD patients can stimulate phoroblasts to secrete MMPs, but its function is partly restrained by IL-21 receptor/ Fc chimera (Stolfi et al., 2011). The stimulation of intestinal epithelial cells by IL-21 may lead to an increase in the phosphorylation of extracellular signal-regulated kinase $1 / 2(E R K 1 / 2)$ and p38. This is compounded by the activation of MIP-3a (a chemical inducer of T cells), which causes T helper 1 cells to induce progressive inflammation. Therefore, the role of IL-21 in IBD may be related to T helper 1 cells (Imaeda et al., 2011). Studies have shown that IL-21 is an important regulatory factor in intestinal inflammation and T helper 17 cell activation, which can result in intestinal damage (Farmer et al., 2011). Lymphocytes are activated by bacterial antigens in the intestinal tract, resulting in the secretion of IL-21. The expression of the IL-21 receptor is also increased in lymphocytes. Combined with the effect of IL-21, the secretion of proinflammatory mediators, such as TNF- $\alpha$ and interferon (IFN)- $-\gamma$, is increased and intestinal mucosal inflammation worsens. Ultimately, this aggravates the intestine further and results in the pathological changes observed in UC (He et al., 2010; Torricelli et al., 2011).

In conclusion, IL-21 may be involved in the early pathological process of ischemiareperfusion injury in the small intestine of rats but the mechanism by which it is involved is not clear. In this study, rats were given a 7\% DSS solution for 14 days, which successfully established a chemically-induced UC model. In addition, the increase in IL-21 in the DSS group compared to the control group was detected in serum and tissue from the small intestine and colon. Blocking the IL-21 pathway may be a new therapeutic scheme for UC. In future studies, we will focus on the specific effect of IL-21 and its relationship with other cytokines in DSS-induced UC.

\section{Conflicts of interest}

The authors declare no conflict of interest.

\section{REFERENCES}

Abromson-Leeman S, Bronson RT and Dorf ME (2009). Encephalitogenic T cells that stably express both T-bet and ROR gamma t consistently produce IFNgamma but have a spectrum of IL-17 profiles. J. Neuroimmunol. 215: 10-24.

Caruso R, Botti E, Sarra M, Esposito M, et al. (2009). Involvement of interleukin-21 in the epidermal hyperplasia of psoriasis. Nat. Med. 15: 1013-1015.

Coquet JM, Kyparissoudis K, Pellicci DG, Besra G, et al. (2007). IL-21 is produced by NKT cells and modulates NKT cell activation and cytokine production. J. Immunol. 178: 2827-2834. 
Ettinger R, Kuchen S and Lipsky PE (2008). The role of IL-21 in regulating B-cell function in health and disease. Immunol. Rev. 223: 60-86.

Farmer DG, Ke B, Shen XD, Kaldas FM, et al. (2011). Interleukin-13 protects mouse intestine from ischemia and reperfusion injury through regulation of innate and adaptive immunity. Transplantation 91: 737-743.

Fina D, Sarra M, Fantini MC, Rizzo A, et al. (2008). Regulation of gut inflammation and th17 cell response by interleukin-21. Gastroenterology 134: 1038-1048.

He Y, Li CM, Hou FX and Liu YJ (2010). [Expression of IL-17 type cytokine and IFN-y type cytokine in ulcerative colitis patients]. China J. Mod. Med. 17: 36-37. (In Chinese)

lannello A, Boulassel MR, Samarani S, Debbeche O, et al. (2010). Dynamics and consequences of IL-21 production in HIVinfected individuals: a longitudinal and cross-sectional study. J. Immunol. 184: 114-126.

Imaeda H, Andoh A, Aomatsu T, Uchiyama K, et al. (2011). Interleukin-33 suppresses Notch ligand expression and prevents goblet cell depletion in dextran sulfate sodium-induced colitis. Int. J. Mol. Med. 28: 573-578.

Monteleone I, Sarra M, Del Vecchio Blanco G, Paoluzi OA, et al. (2010). Characterization of IL-17A-producing cells in celiac disease mucosa. J. Immunol. 184: 2211-2218.

Park SW, Chen SW, Kim M, Brown KM, et al. (2011). Cytokines induce small intestine and liver injury after renal ischemia or nephrectomy. Lab Invest. 91: 63-84.

Sarra M, Monteleone I, Stolfi C, Fantini MC, et al. (2010). Interferon-gamma-expressing cells are a major source of interleukin-21 in inflammatory bowel diseases. Inflamm. Bowel Dis. 16: 1332-1339.

Shichita T, Sugiyama Y, Ooboshi H, Sugimori H, et al. (2009). Pivotal role of cerebral interleukin-17-producing gammadeltaT cells in the delayed phase of ischemic brain injury. Nat. Med. 15: 946-950.

Snoeijs MG, Van Bijnen A, Swennen E, Haenen GR, et al. (2011). Tubular epithelial injury and inflammation after ischemia and reperfusion in human kidney transplantation. Ann. Surg. 253: 598-604.

Spolski R and Leonard WJ (2008). Interleukin-21: basic biology and implications for cancer and autoimmunity. Annu. Rev. Immunol. 26: 57-79.

Stolfi C, Rizzo A, Franzè E, Rotondi A, et al. (2011). Involvement of interleukin-21 in the regulation of colitis-associated colon cancer. J. Exp. Med. 208: 2279-2290.

Torricelli M, Bellisai F, Novembri R, Galeazzi LR, et al. (2011). High levels of maternal serum IL-17 and activin A in pregnant women affected by systemic lupus erythematosus. Am. J. Reprod. Immunol. 66: 84-89.

Vogelzang A, McGuire HM, Yu D, Sprent J, et al. (2008). A fundamental role for interleukin-21 in the generation of T follicular helper cells. Immunity 29: 127-137.

Xia XZ and Li ZJ (2009). IL-21 receptor is highly expressed and induces proinflammatory cytokine secretion in patients with ulcerative colitis. World Chin. J. Digestol. 17: 102-105.

Zhu X, Ma D, Zhang J, Peng J, et al. (2010). Elevated interleukin-21 correlated to Th17 and Th1cells in patients with immune thrombocytopenia. J. Clin. Immunol. 30: 253-259. 